\title{
Pattern of Upper Aerodigestive Tract Malignancies and Metastatic Lymph Nodes
}

\author{
Dr Ajaykumar .K .F ${ }^{1}$, Dr Pramod Menon ${ }^{2}$ \\ ${ }^{1}$ Assistant Professor of ENT, Govt.Medical College, Thrissur, Kerala \\ ${ }^{2}$ Additional Professor of ENT, Govt.Medical College, Thrissur, Kerala
}

\begin{abstract}
The status of the regional lymphatics in head and neck malignancy is one of the most important prognostic indicators. This study included 50 patientsattending the department of ENT, Medical College, during a period of one year from June 2010. We found that the maximumnumbers of cases were of carcinoma hypopharynx, 20 cases (40\%), followed by carcinoma oropharynx, 14 cases (28\%).The commonest site of metastases from all upper aerodigestive tract tumours is to the ipsilateral L II nodes.
\end{abstract}

Keywords: Metastatic Neck Nodes, Upper Aerodigestive Tract Malignancy, Nodal Staging, Tumour Staging

\section{Introduction}

Head and neck cancer ranks as the sixth mostcommon cancer worldwide. 1 thus making head and neck cancer a major health problem. Over 90 percent of these aresquamous carcinomas arising from the epithelium of the upper aerodigestive tract (oral cavity, oropharynx, nasopharynx,hypopharynx and larynx). The status of the regional lymphatics is one of the most important prognostic indicators in patients with head and neck cancer. The presence of regional metastases results in cure rates that are approximately half of those obtainable if metastasis to the regional lymphatics is not present. Thus the treatment of the neck has become one of the most actively debated topics in the field of headand neck oncology. The concept that squamous cell carcinoma of the head and neck metastasizes to regional lymph nodes in a predictable distribution has become generally accepted. Clinical application of this concept has decreased the morbidity of treatment through selective modifications of the radical neck dissection and through more accurate application of irradiation to target the nodal basins at risk. Appropriate application of this more selective treatment requires an understanding of the patterns of lymphatic drainage to the neck.

The aim of this study is to assess the patterns of cervical lymph node metastasis from various primary squamous cell carcinomas of the upper aerodigestivetract, which will help in the management of our patients with upper aerodigestive tract malignancies.

\section{Materials and Methods}

This is a cross sectional study of fifty previously untreated patients, presenting with features of upper aerodigestive tract malignancies with metastatic neck nodes, attending the department of ENT, Medical College, during a period of one year from June 2010.A detailed history and physical examination was done, which included examination of the neck for assessment of the neck nodes and a full otorhinolaryngological examination to identify and assess the primary in the upper aerodigestive tract. After the necessary investigations, these patients were subjected to Fine Needle Aspiration Cytology (FNAC) of the neck nodes and open biopsy of lymph nodes, if FNAC is inconclusive. These patients were also subjected to upper aerodigestive tract rigid endoscopy for proper assessment and biopsy of the primary. Patients with isolated supraclavicular lymph nodes and Level VI nodes were excluded as majority of the primary were found to be located below the clavicle and Level VI nodal involvement are mainly from a primary in the thyroid gland.

\section{Observation and Results}

This study included total of 50 patients, with 45 males and 5 females. Age range was from 24 years to 84 years.

Six major sites were involved in our study. Maximum number of cases was of carcinoma hypopharynx, 20 cases (40\%), followed by carcinoma oropharynx, 14 cases $(28 \%)$ and the remaining were supraglottic, 8 cases $(16 \%)$, nasopharynx, 4 cases $(8 \%)$ oral cavity, 3 cases $(6 \%)$ and glottic malignancy one case $(2 \%)$ respectively as shown in figure1

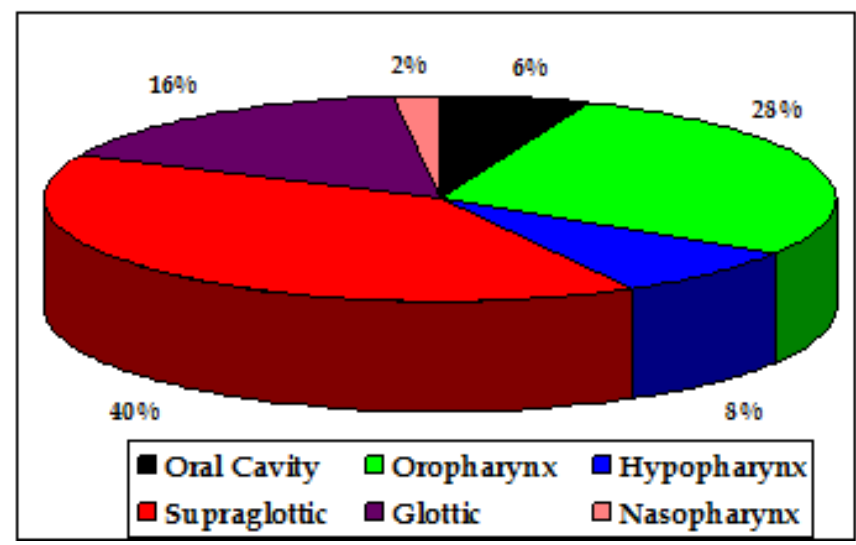

Figure 1: Percentage of Different Malignancies

No patient had involvement of lymph nodes in all the five neck levels. Interestingly only two patients had involvement of four neck levels from L II to L V. Both cases were nasopharyngeal malignancies. Isolated $\mathrm{L} \mathrm{V}$ involvement was noticed in 4 cases of that three patients had hypopharyngeal malignancy and one had supraglottic malignancy. Among this, one case of hypopharyngeal malignancy had both 


\section{International Journal of Science and Research (IJSR) \\ ISSN (Online): 2319-7064}

Index Copernicus Value (2015): 78.96 | Impact Factor (2015): 6.391

ipsilateral and contralateral $\mathrm{L} \mathrm{V}$ level involvement without the involvement of other neck levels. IpsilateralL IV alone was involved in one case of oropharyngeal malignancy Majority of cases $26(52 \%)$ had involvement at one level only, of that majority were at L II in 17 patients followed by L III in 4 cases and L V in 3 and one each in L I and L IV. Bilateral L III involvement was seen in one case of hypopharyngeal malignancy,two cases of hypopharyngeal malignancy.

Regarding the nodal staging (Table 1 and figure 2) 20 cases $(40 \%)$ were in N1 nodal staging followed by N2c in 13 $(26 \%)$ cases and N2b in 7(14\%). 3 patients each had N2a and N3 nodal staging. Next we consider nasopharyngeal malignancy in which nodal staging is slightly different. Majority of cases belong to N2 in $3(75 \%)$ cases and one belongs to N3a.

Table 1

\begin{tabular}{|l|l|l|l|l|l|l|}
\hline Malignancy & N1 & N2a & N2b & N2c & N3 & Total \\
\hline Oral Cavity & 2 & 0 & 0 & 1 & 0 & 3 \\
\hline Oropharynx & 7 & 0 & 1 & 5 & 1 & 14 \\
\hline Hypopharynx & 7 & 1 & 5 & 6 & 1 & 20 \\
\hline Supraglottic & 3 & 2 & 1 & 1 & 1 & 8 \\
\hline Glottic & 1 & 0 & 0 & 0 & 0 & 1 \\
\hline Nasopharynx & 0 & 3 & 0 & 0 & 1 & 4 \\
\hline
\end{tabular}

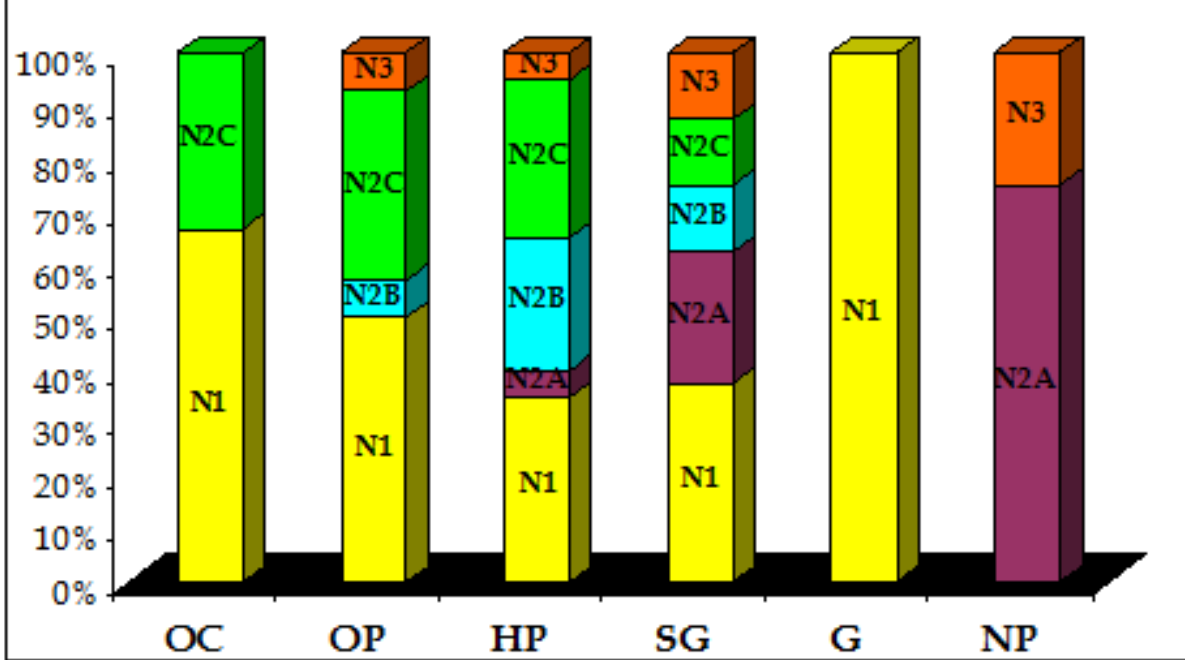

Figure 2: Percentage Wise Distribution of Nodal Staging

When we correlate tumour staging with nodal staging (Table-2), in the case of carcinoma oral cavity one had $\mathrm{T} 2 \mathrm{~N} 1$, one had T2N2c and one had T3N1. So the higher nodal staging is not correlated with higher tumour staging. When we consider oropharyngeal malignancy one case was in T1N1, 5 in $\mathrm{T} 2 \mathrm{~N} 1$, two in $\mathrm{T} 2 \mathrm{~N} 2 \mathrm{c}$, one in $\mathrm{T} 3 \mathrm{~N} 1$, one in $\mathrm{T} 3 \mathrm{~N} 2 \mathrm{~b}, 3$ in $\mathrm{T} 3 \mathrm{~N} 2 \mathrm{c}$ and one in T3N3. Here higher nodal staging is seen with higher tumour staging. In the case of hypopharyngeal malignancy, one had $\mathrm{T} 1 \mathrm{~N} 2 \mathrm{~b}$, one had T1N2c, three had T2N1, two had T2N2c, 4 had T3N1, one had T3N2a, 4 had T3N2b, three had T3N2c and one had T3N3. Here also we can see that higher nodal staging were seen with higher tumour staging. But lower nodal staging (N1) is not seen in T1 lesions but seen in T2 and T3 In the case of supraglottic lesions one had T1N1, two had T2N2a, two had T3N1 and one each had T3N2b, T3N2c and T3N3. Here also higher nodal staging are seen with higher tumour staging. The only case glottis malignancy belongs to T3N1. In the case of nasopharyngeal malignancy two cases in T1N2 and one case in T1N3A and one in T2N2. Here higher nodal staging (N3A) was seen in T1 lesion. Here also higher nodal staging is not seen with higher tumour staging. Overall size of the lymph node (in greatest dimension) varied from $1.5 \mathrm{~cm}$ to $12 \mathrm{~cm}$.
Table 2

\begin{tabular}{|c|c|c|c|c|c|c|c|}
\hline $\begin{array}{c}\text { Type of } \\
\text { Malignancy }\end{array}$ & Tumour & N1 & $\mathrm{N} 2 \mathrm{a}$ & $\mathrm{N} 2 \mathrm{~b}$ & N2c & N3 & Total \\
\hline \multirow{3}{*}{ Oral Cavity } & $\mathrm{T} 1$ & & & & & & 0 \\
\hline & $\mathrm{T} 2$ & 1 & & & 1 & & 2 \\
\hline & T3 & 1 & & & & & 1 \\
\hline \multirow{3}{*}{ Oropharynx } & T1 & 1 & & & & & 1 \\
\hline & $\mathrm{T} 2$ & 5 & & & 2 & & 7 \\
\hline & T3 & 1 & & 1 & 3 & 1 & 6 \\
\hline \multirow{3}{*}{ Hypopharynx } & T1 & & & 1 & 1 & & 2 \\
\hline & $\mathrm{T} 2$ & 3 & & & 2 & & 5 \\
\hline & T3 & 4 & & 4 & 3 & 1 & 12 \\
\hline \multirow{3}{*}{ Supraglottic } & T1 & 1 & & & & & 1 \\
\hline & $\mathrm{T} 2$ & & & & & & 0 \\
\hline & T3 & 2 & & 1 & 1 & 1 & 5 \\
\hline \multirow{4}{*}{ Glottic } & T1 & & & & & & 0 \\
\hline & $\mathrm{T} 2$ & & & & & & 0 \\
\hline & T3 & 1 & & & & & 1 \\
\hline & Tumour & N1 & $\mathrm{N} 2$ & N3a & N3b & & Total \\
\hline \multirow{3}{*}{ Nasopharynx } & $\mathrm{T} 1$ & & 2 & 1 & & & 3 \\
\hline & $\mathrm{T} 2$ & & 1 & & & & 1 \\
\hline & T3 & & & & & & 0 \\
\hline
\end{tabular}

\section{Discussion}

Regarding the size of primary (tumour staging)most of the cases were in the T3(52\%) followed by T2(34\%)and $\mathrm{T} 1(14 \%)$.In the case of oral cavity $(67 \%)$ and oropharynx $(50 \%), \mathrm{T} 2$ was the predominant tumourstage. In the case of 


\section{International Journal of Science and Research (IJSR) \\ ISSN (Online): 2319-7064}

Index Copernicus Value (2015): 78.96 | Impact Factor (2015): 6.391

hypopharynx (65\%), supraglottis (63\%) and glottis ,T3 was the predominant tumour stage and $\mathrm{T} 1$ was predominating in the case of nasopharynx (75\%). When we consider total number of patients with $\mathrm{T} 1$ stage, majority was nasopharyngeal (3/7).In the case of T2 majority was oropharynx(7/17). In the case of T3 majority was in hypopharynx(13/26). Hence we can conclude that nasopharyngeal carcinomas are developing lymph node metastasis in early tumour staging and hypopharynx and larynx malignancies metastasize in late tumour staging.

Regarding the pattern of cervical lymph node metastasis from the upper aerodigestive tract malignancies, overallipsilateral LII neck level was predominantly involved followed by ipsilateral LIII and L V. In the case of oral cavity malignancy L I was predominantly involved followed by LII and LIII.LIV and LV were not involved.This is consistent with the classical study by Lindberg[2], even though he has described the oral cavity malignancy under different headings.It is also consistent with the study of of Shah et al[3] and also with Li et al[4], even though L IV and $\mathrm{L}$ Vinvolvement were noticed in few patients in these two studies.In study of Shah et al, in which they studied the metastasis from squamous cell carcinoma of oral cavity only.In their study L I was seen in $48 \%$, L II in $38 \%$ and L III in $31 \%$ which was comparable to our study eventhough number of patients with oral cavity was less in our study.We had 66\% L I,33\% LII and 33\% LIII(ipsilateral).

In the case of hypopharynxipsilateral L II, was predominantly involved $(75 \%)$ followed by ipsilateral L III (40\%) and L V (25\%).Ipsilateral L I and L IV was involved in 5\%.In the Lindberg`s[2] study, ipsilateral LII (73\%) was predominant followed by ipsilateral L III $(55 \%)$, Ipsilateral L IV (21\%),ipsilateral L V in $14 \%$ and ipsilateral L I was involved in only $1 \%$.So the difference is that in our study, the order of predominance was in ipsilateral L II, L III and L $\mathrm{V}$ followed by L I and L IV.

In the case of supraglottic malignancy ipsilateral L II was predominantly involved( $75 \%$ ) followed by ipsilateral L III (38\%) and ipsilateral L V and contralateral L II (13\% each). No L I and L IV involvement was noticed.In the case of Lindberg study the order of involvement was ipsilateral L II (67\%), ipsilateral L III $(48 \%)$, contralateral L II (21\%)ipsilateral L IV in $15 \%$, contralateral L III in $10 \%$,ipsilateral L V in $9 \%$. L I was involved in only $2 \%$ of cases in Lindberg's study. In our study we had more cases of L V than L IV and contralateral nodal involvement was less in our study compared to that of Lindberg.

We had only one case of glottis malignancy and that was only ipsilateral LIIinvolvement.In the Lindberg's study glottis malignancy was not reported. In the study by Li et al[4],they have reported the cases as malignancy larynx with L II, L III and L IV predominating.Byres et al[5] has reported the glottis malignancy with ipsilateral LII involvement in $55 \%$ and L III in $27 \%$ with no L I, L IV and $\mathrm{L} \mathrm{V}$ involvement.

$52 \%$ of cases had metastasis only at one level with ipsilateral L I (2\%) ipsilateral L II (34\%)ipsilateralL III (8\%)ipsilateral L IV (2\%) and ipsilateralL V (6\%).Total percentage was comparable with the study of Li et al[4], but isolated L V was not reported by Li et al. Isolated L V involvement were seen in one case of supraglottis and two cases of hypopharynx.One hypopharyngeal malignancy had bilateral $\mathrm{L} \mathrm{V}$ involvement without involvement of other groups.So this finding is very important because in the neck where lymphatics are particularly profuse, the systematic spread of cancer from one lymph node area to other does not occur and metastases may jump to a more distant node even in the absence of previous treatment.(6,7).This is one of the anxieties regarding selective neck dissection. We also had two cases of ipsilateral L II, L III and L V involvement without L IV and two cases of L II and L V involvement alone.One case of nasopharyngeal carcinoma had ipsilateral L II, L III, L V and contralateral L IV.

In our study we had higher incidence of $\mathrm{L} \mathrm{V}$ involvement (overall 28\%).Isolated $\mathrm{L} \mathrm{V}$ involvement was seen in $8 \%$ of cases. L V involvement was more common in nasopharyngeal and hypopharyngeal carcinoma.In the study by Davidson and colleagues [8], L V involvement was noticed in only $3 \%$ of cases, highest in patients with hypopharyngeal and oropharyngeal primary sites $(7 \%$ and $6 \%$ respectively).

When we consider the overall nodal staging $\mathrm{N} 1$ was predominating $(40 \%)$ followed by $\mathrm{N} 2 \mathrm{c}(26 \%)$ and $\mathrm{N} 2 \mathrm{~b}(14 \%)$ and $\mathrm{N} 2 \mathrm{a}$ and $\mathrm{N} 3$ in $6 \%$ each.In the case of oral cavity N1 was predominating.In the case of oropharynx, hypopharynx, supraglottis and glottis also N1 was predominating.But in the case of nasopharynx,N2 was predominating $(75 \%)$.

When we correlate with the tumour staging and nodal staging in each primary sites,in the case of oral cavity higher nodal staging was not found with higher tumour staging.N2c was seen with T2 lesion and one patient with T3 lesion had only N1 involvement.In the case of oropharynx higher nodal staging was seen with higher tumourstaging. One N3, nodal staging was seen with T3 lesion and N2c involvement in 3 cases of T3 and 2 cases of T2. T1 had only N1 involvement.In the case of hypopharynx also higher nodal involvement was found with higher tumour staging. But more N1 involvement are also seen with T3.In the case of supraglottis also higher nodal staging are seen with higher tumour staging but more N1 involvement are seen with T3 lesions. In the case of nasopharynx, higher nodal involvement (N3a)are found with $\mathrm{T} 1$ and $\mathrm{T} 2$ lesion had only N2 involvement. So in addition to the tumour staging there may be some other factors also that affects the nodal staging. Similar findings were also reported by Lindberg [2] and he attributed it to the aggressiveness of the primary.

\section{Conclusion}

The status of the regional lymphatics in head and neck malignancy is one of the most important prognostic indicators.Metastatic cervical lymphadenopathy from squamous cell carcinoma of the upper aerodigestive tract has a predictable pattern. The commonest site of metastases from all upper aerodigestive tract tumours is to the ipsilateral L II nodes. Neck levels L I,LII,L III(ipsilateral) were at greatest risk for nodal metastases from primary 


\section{International Journal of Science and Research (IJSR) \\ ISSN (Online): 2319-7064}

Index Copernicus Value (2015): 78.96 | Impact Factor (2015): 6.391

squamous cell carcinoma of oral cavity.L IV and L V involvement was rare.

Tumours of the nasopharynx are unique among squamous cell carcinoma of the upper aerodigestive tract in thatthey commonly metastasize to L II and L V.

Level $\mathrm{V}$ metastasis is not uncommon in upper aerodigestive tract malignancies. Isolated $\mathrm{L} \mathrm{V}$ involvement was also seen in $8 \%$ of cases.

\section{References}

[1] FerlitoA, RinaldoA, DevaneyKo, et al. Prognostic significance of microscopic and macroscopic capsular spread from metastatic tumour in the cervical lymph nodes.OralOncol 2002;38:747-51.

[2] Lindberg R: Distribution of cervical lymph node metastases from squamous cell carcinoma of the upper respiratory and digestive tracts. Cancer 1972; 29:1446.

[3] Shah JP, Candela FC, Poddar AK. The patterns of cervicaloral cavity. Cancer 1990;66:109-13..

[4] Xiao M1ngLi., William IgnaceWei.Cervical lymph node metastatic patterns of squamous carcinomas in the upper aerodigestive tract. J L O 1996; 110 ; 937941

[5] Byers RM, Wolf PF, Ballantyne AJ. Rationale for modifiedneck dissection. Head Neck Surg 1988;10:1607.

[6] Heimlich HJ.Carcinoma of the cervical oesophagus.Journal of Thoracic and Cardiovascular surgery. 1970;59:309-18.

[7] FutrellJW,BennettSH,HoyeRC,RothJA,KetchamAS.Pre dicting survival in cancer of larynx and hypopharynx. American Journal of Surgery. 1971;122:451-7.

[8] Davidson BJ, Kulkarny V, Delacure MD, Shah JP. Posterior triangle metastases of squamous cell carcinoma of the upper aerodigestive tract. Am J Surg 1993; 166:395-8. 